NASA Technical Memorandum 106972

\title{
Acousto-Ultrasonic Decay in Metal Matrix Composite Panels
}

Harold E. Kautz

Lewis Research Center

Cleveland, Ohio

August 1995

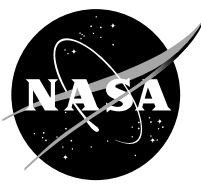

National Aeronautics and

Space Administration 


\title{
ACOUSTO-ULTRASONIC DECAY IN METAL MATRIX COMPOSITE PANELS
}

\author{
Harold E. Kautz \\ National Aeronautics and Space Administration \\ Lewis Research Center \\ Cleveland, $\mathrm{OH} 44135$
}

\begin{abstract}
Acousto-ultrasonic (A-U) decay rates (UD) were measured in metal matrix composite (MMC) panels. The MMC panels had fiber architectures and cross-sectional thicknesses corresponding to those designed for aerospace turbine engine structures. The wavelength-to-thickness ratio produced by the combination of experimental frequency setting conditions and specimen geometry was found to be a key parameter for identifying optimum conditions for UD measurements. The ratio was shown to be a useful rule of thumb when applied to ceramic matrix composites (CMC)s and monolithic thermoplastics.
\end{abstract}

\section{Introduction}

To accomodate materials characterization requirements of ceramic matrix composite (CMC) structures for advanced aircraft engines the ring-down count used in acoustic emission (AE) was combined with diffuse field decay analysis $1-4$. This combination, termed a "pseudo" diffuse field decay was recently shown to be useful in monitoring mechanical degradation in laboratory CMC coupons. 5

The ring-down count is the number of times a resonant or narrow band AE signal exceeds a fixed voltage threshold before it decays away. This can also be be used as a measure of relative attenuation or decay rate of broadband A-U signals. True diffuse field decay depends upon the phases and amplitudes of the ultrasonic propagation modes in the A-U signal becoming totally random during the interval when the signal passes from the sending transducer to the receiver.

The requirements of true diffuse field decay place certain restrictions on the geometry of the part being examined. For useful applications to aerospace structures, the potential of UD for interrogating degradation state recommends the exploration of the degree to which the diffuse field requirements may be relaxed. The present work begins this exploration.

\section{Theory and Experiment}

The method of obtaining UD data from collected A-U signals is detailed elsewhere. ${ }^{1-3}$ One induces an exponentially decaying signal to arrive at the receiver. This is collected and analyzed. Comments on strategies for this 
analysis can be found in the appendix. It may happen that the presence of nondispersive - or nearly nondispersive - plate modes will produce components in the signal with well defined velocity and phase. ${ }^{6,7}$ This is undesireable for the present application. One may be able to avoid this by frequency filtering or by using only a segment of the time domain. In the end, it may be satisfactory that the nondispersive portion be small. Once this is determined, the time domain is partitioned. These partitions are Fourier transformed and the power spectrum is regressed logarithmicly against the center values of the partition times. The slope of this regression is the UD rate.

UD rates are frequency dependent because they derive from ultrasonic attenuation. In cases where the ultrasonic velocity is well defined attenuation can be calculated from UD rate. However, waveforms collected in the A-U configuration are often dispersive. It may be possible to filter the power spectrum to yield UD as a function of frequency. Other times, experimental conditions may limit the portions of the spectrum that have sufficient magnitude to yield accurate values.

In this study A-U waveforms were collected on two SCS-6/Ti 15-3 unidirectional panels. One was considered a "thin" plate, the other a "thick" plate. Experimental conditions were compared to those realized in measurements on CMCs. Since we deal with plate waves in most cases, a likely matching condition is the nondimentional ratio of wave length, $\lambda$, to plate thickness, h:

$$
\text { Ratio }=\lambda / \mathrm{h}
$$

In terms of readily measureable quantities this can be expressed as:

$$
\lambda / \mathrm{h}=\mathrm{V} / \mathrm{fh}
$$

$\mathrm{V}$ is the axial velocity in the thickness direction. $\mathrm{f}$ is the frequency at which the ratio is being evaluated.

Table I lists the specimens used in this investigation. The CMC had geometry typical of the work cited earlier. A-U signals were collected with pairs broad band piezoelectric transducers with nominal center frequencies 0.2 , 0.5, 1.0, 2.25, 5.0 and 10.0 MHz. Elastomer pads were used as the coupling medium except with the $10.0 \mathrm{MHz}$ pair. In this case a gel couplant was used. Transducer center line separation was maintained at $3.8 \mathrm{~cm}$ in all of the present work.

\section{Results and Discussion}

\section{Comparing A-U Waveforms Between the Materials}

Figure 1 shows data collected with $0.5 \mathrm{MHz}$ on the thin MMC panel. For this case the wavelength ratio is found to be:

$$
\lambda / \mathrm{h}=9.6
$$

This is in the regime where lowest antisymmetric mode and lowest symmetric mode pulses are dominate, allowing determination of the velocities VS and VL, respectively. 3,7 Figure 2 shows the same regime for a CMC specimen with $0.5 \mathrm{MHz}$ transducers. For the conditions in Fig. 2: 


$$
\lambda / \mathrm{h}=8.1
$$

Figures 1 and 2 are similar although they represent data from two different materials. The first promiment pulse, (designated 1) in each figure is due to the lowest antisymmetric plate mode. In each figure pulse 1 has passed directly from sender to receiver without any edge reflections. Subsequent pulses are the same lowest antisymmetric mode after reflections off of edges. There arrival time at the receiver is dependent on the geometry of the specimen and the placement of the transducers relative to the edges. These later pulses can be traced by touching a wet finger to the specimen beyond the transducer spacing on either end. The later pulses are diminished by this act, but the first is uneffected.

The pulse designated 2 in Figs. 1 and 2 is the direct path arrival of the first symmetric mode at the receiver. This pulse always is smaller than pulse 1 .

In order to apply this regime to the thick MMC panel it was necessary to employe $0.2 \mathrm{MHz}$ transducers. Figures 3 shows the A-U waveform collected. For figure 3:

$$
\lambda / \mathrm{h}=7.9
$$

This waveform is similar to those of figures 1 and 2 if one takes into account its broader period of oscillation. The actual collected waveform is centered at about .11 MHz, which is a factor of 4 lower than in Figs. 1 and 2 . The time domain pulse is about 4 times as wide leading to some overlap.

Figure 4 is an example of an A-U waveform collected on the thick MMC panel when $0.5 \mathrm{MHz}$ transducers are employed. In this case:

$$
\lambda / \mathrm{h}=2.3
$$

Figure 5 shows a CMC using 2.25 MHz transducers, with:

$$
\lambda / \mathrm{h}=1.9
$$

Figure 6 shows the thin MMC using 2.25 MHz transducers, with:

$$
\lambda / \mathrm{h}=2.1
$$

The A-U waveforms in figures 4, 5, and 6 are similar to each other in terms of displaying an exponential decay. This is a desireable condition for determining UD rates. This is a different regime from that encountered in Figs. 1 to 3. Pulse arrivals appear much more frequently here. Since the lowest antisymmetric pulse does not travel fast enough to return to the receiver this frequently, it clear that higher plate modes are now being excited. They are very disperse and travel much slower. Plate wave dispersion diagrams reveal this. 6,7 A collection of these higher plate mode arrivals has the randomness in phase and amplitude that is desireable for UD.

The waveforms of Figs. 7 and 8 were collected under conditions of much smaller wavelength to thickness ratios. Figure 7 is from the thick MMC with $10.0 \mathrm{MHz}$ transducers. For Fig. 7:

$$
\lambda / \mathrm{h}=0.11
$$


Figure 8 is for a thermoplastic panel with $2.25 \mathrm{MHz}$ transducers:

$$
\lambda / \mathrm{h}=0.12
$$

In both Figs. 7 and 8 the ratio is sufficiently low so that propagation paths of the original sender pulse arrive discretely at the receiver. This is a third $\lambda / \mathrm{h}$ regime. It is one where, at least in monolithic materials, individual propagation paths can be identified. The analysis by propagation paths has been worked out by Williams et al. ${ }^{8}$ and used later to study interlaminar shear strength in filament wound composites. 9

Three wavelength to thickness ratio regimes have been demonstrated:

Regime 1 contains $\lambda / \mathrm{h}$ values in the vicinity of 8 . The lowest symmetric and antisymmetric plate modes dominate the waveform.

Regime 2 contains the $\lambda / \mathrm{h}$ value in the vicinity of 2 . The waveform is dominated by an exponential decay. This is the most desireable region for determining UD rate.

Regime 3 contains $\lambda / \mathrm{h}$ values in the vicinity of 0.1 . Here Ultrasonic pulse paths arrive at the receiver discretely.

\section{Determining ultrasonic decay rates in the MMCs}

It may happen that experimental or geometrical conditions will preclude measurements in the optimum UD regime. (This could happen, for example, if one wishes to interrogate an engine component). For this reason, it is of interest to examine the possibility of extending the regime. It is also of interest to examine whether decay rate is dependent on thickness. Figure 9 exhibits UD rates for the SCS-6/Ti 15-3 unidirectional panels where both $\lambda / \mathrm{h}$ and h are varied. These rates are plotted as a function of the center frequency of the spectra. Only data collected for propagation path parallel to the fiber direction are shown.

Note that the UD rates for the thin and the thick panel fall on the same curve as a function of center frequency of the power spectrum filter. This implies that decay rates determined are a function of the material and not of its geometry. In the case of the thin plate, results are plotted at $0.4,0.8$, and $2.0 \mathrm{MHz}$. These correspond, respectively, to regime 1 (exhibited in figure 1), a value intermediate between regimes 1 and 2, and regime 2 (exhibited in Fig. 6). In order to get the thin panel regime 1 data to fit on the curve it was necessary to window out the lowest antisymmetric plate wave pulses prior to calculating the power spectra. When this plate wave pulse is left in, the UD rate at $0.4 \mathrm{MHz}$ is the highest of the three decay rates rather than the lowest. A departure of the (non-windowed) UD rate from the curve at low frequency has been noted elsewhere. 1

For the thick panel, the $0.4 \mathrm{MHz}$ data point is in regime 2 (figure 4). From Fig. 7 note that the $7.5 \mathrm{MHz}$ data points are well into regime 3 . In regime 3 the power spectra are determined for time segments that are dominated by arrivals of multipli-reflecting individual propagation paths. 
Figure 10 shows decay rates as a function of propagation direction with respect to fiber orientation for the SCS-6/Ti 15-3. Below $2 \mathrm{MHz}$ the data from the thin panel is used and above $2 \mathrm{MHz}$ the thick panel. There is a slight indication at higher frequency that decay rate parallel to the fiber direction is greater than at 90 degrees to it. These differences are not conclusive, however.

Data was also taken in diagonal orientation, (ie. $45^{\circ}$ to fiber direction). This data had much greater scatter than did the $0^{\circ}$ or $90^{\circ}$ data. It is likely that this assymmetry accross the propagation path tended to deflect the ultrasound away from the receiver in a non-uniform way.

Although the present data shows little UD dependence on fiber orientation in MMCs, degradation studies may reveal deterioration of fiber/matrix interface and generate orientation effects.

\section{Conclusions}

The technique of matching the wavelength to thickness ratio condition found successful in CMCs is useful for identifying conditions for UD measurements in two thicknesses of SCS-6/Ti 15-3 MMCs. Measurements with very low values of wavelength to thickness ratio (regime 3) suggests that the technique can be extended to much thicker geometries of MMCs such aerospace components.

The motivation for these experiments with MMCs was the success UD has shown in sensing degradation in CMCs. It has not so far been demonstrated whether the technique is similarly sensitive to degradation in MMCs. It is therefore desireable to study the effect of damage on UD state for MMCs.

Table I.- SPECIMENS STUDIED

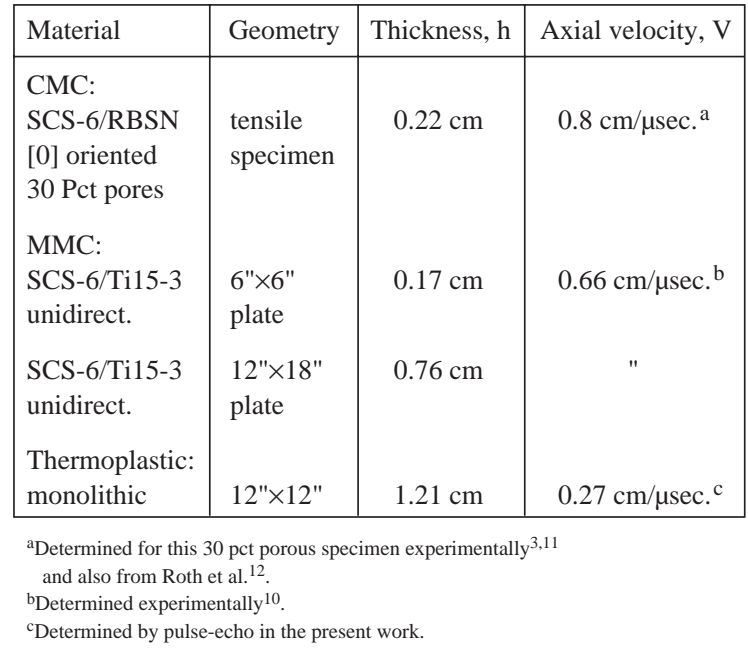




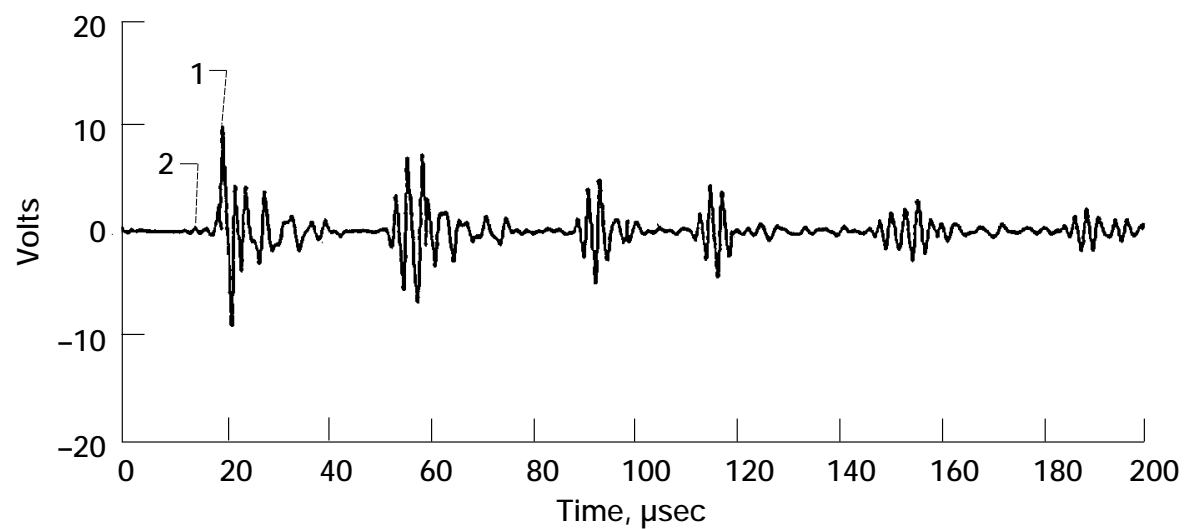

Figure 1.-An acousto-ultrasonic waveform collected on: thin unidirectional SCS-6/Ti 15-3 with $\lambda / \mathrm{h}=9.6,0.5 \mathrm{MHz}$ broad band transducers, wave path [0].

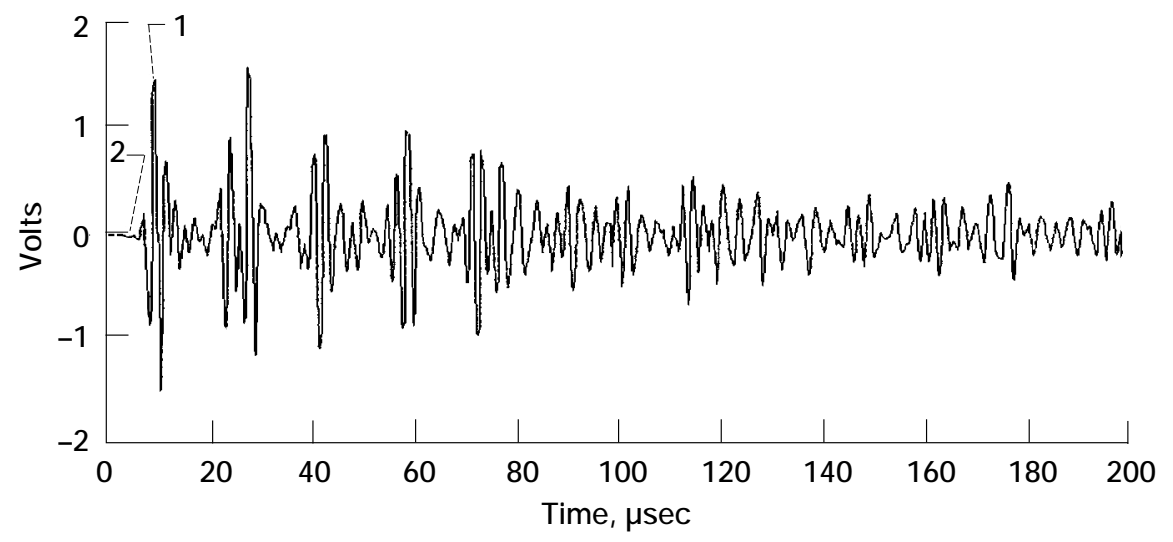

Figure 2.-An acousto-ultrasonic waveform collected on: unidirectional SCS-6/RBSN CMC with $\boldsymbol{\lambda} / \mathrm{h}=8.1$ with: $0.5 \mathrm{MHz}$ broad band transducers, wave path [0].

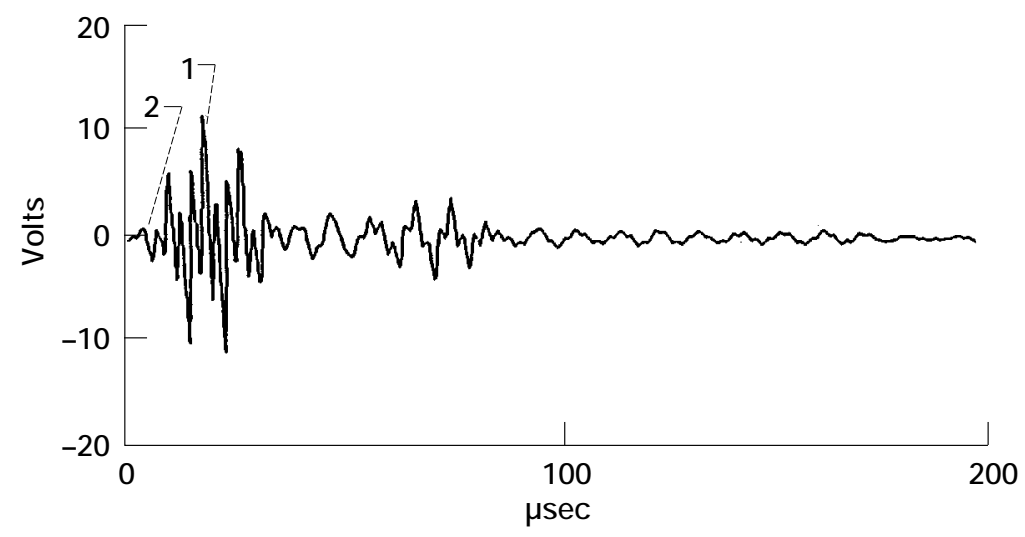

Figure 3.-An acousto-ultrasonic waveform collected on: thick undirectional SCS-6/Ti 15-3 with $\lambda / \mathrm{h}=7.9,0.2 \mathrm{MHz}$ broad band transducers, wave path [0]. 


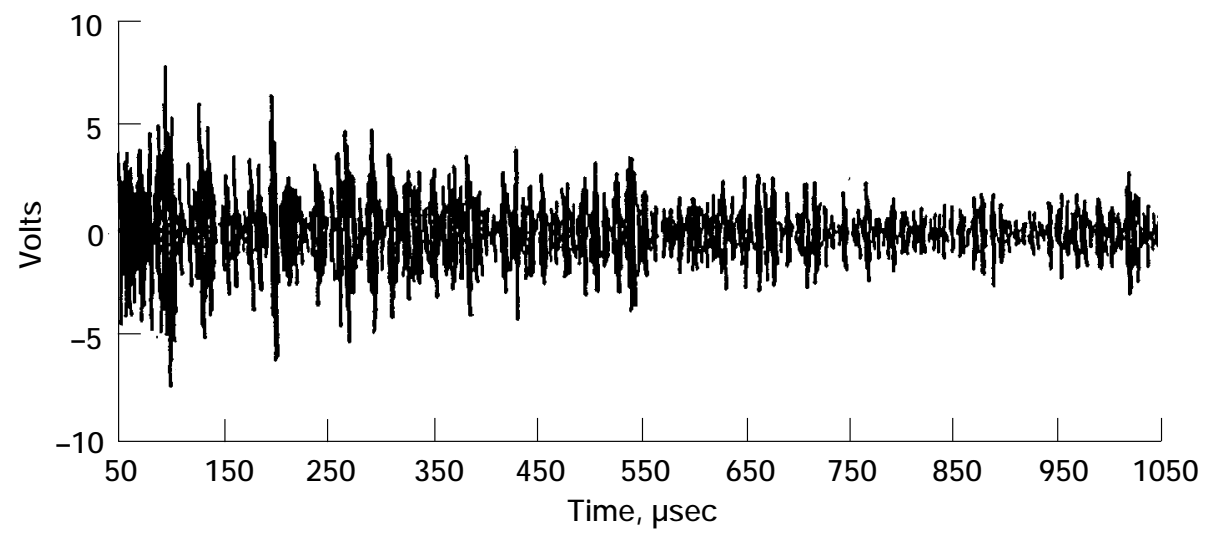

Figure 4.-An acousto-ultrasonic waveform collected on: thick unidirectional SCS-6/Ti 15-3 with $\lambda / \mathrm{h}=2.3,0.5 \mathrm{MHz}$ broad band transducers, wave path [0].

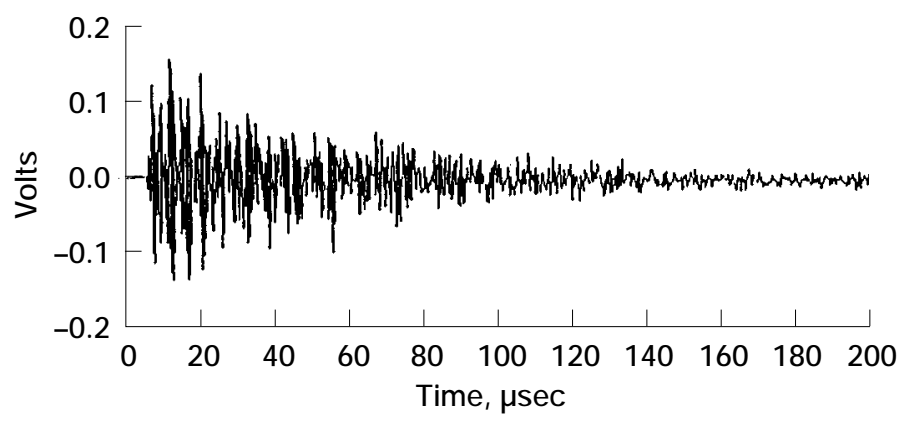

Figure 5.-An acousto-ultrasonic waveform collected on unidirectional SCS-6/RBSN CMC with $\lambda / h=1.9$ with: $2.25 \mathrm{MHz}$ broad band transducers, wave path [0].

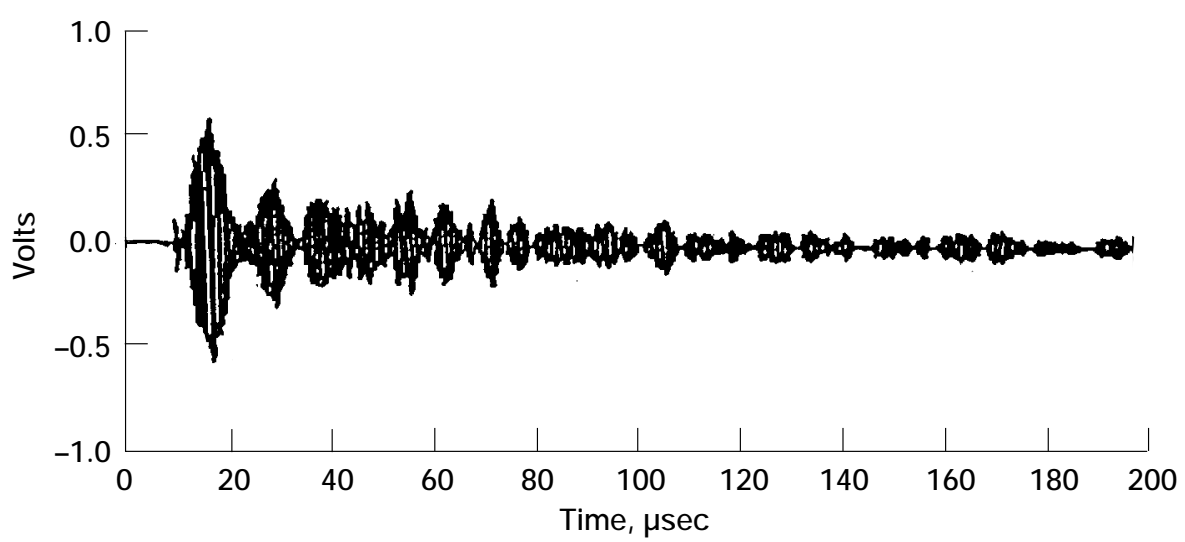

Figure 6.-An acousto-ultrasonic waveform collected on: Thin unidirectional SCS-6/Ti 15-3 with $\lambda / \mathrm{h}=2.1,2.25 \mathrm{MHz}$ broad band transducers, wave path [0]. 


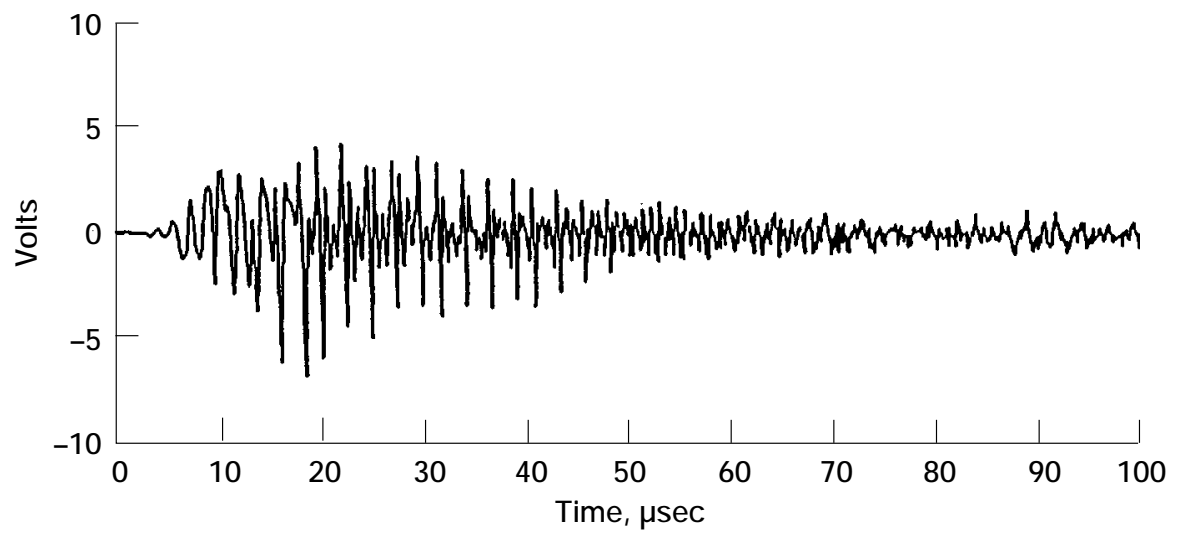

Figure 7.-An acousto-ultrasonic waveform collected on: thick unidirectional SCS-6 /Ti 15-3 with $\lambda / \mathrm{h}=0.11,10.0 \mathrm{MHz}$ broad band transducers, wave path [0].

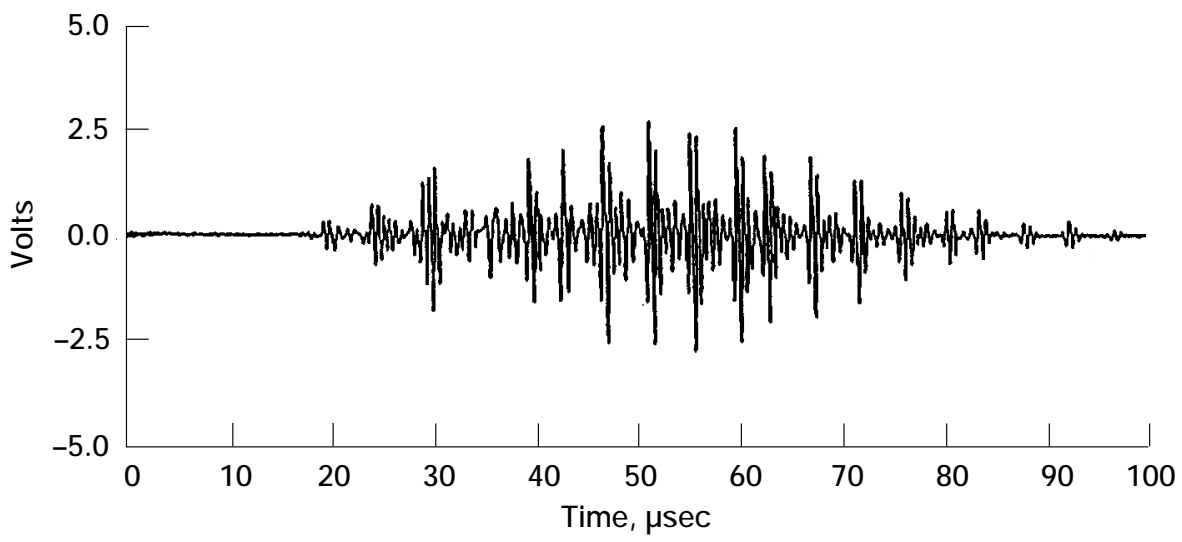

Figure 8.-An acousto-ultrasonic waveform collected on: thermoplastic panel, $\mathrm{h}=1.21 \mathrm{~cm}$ with $\mathrm{\lambda} / \mathrm{h}=0.12,2.25 \mathrm{MHz}$ broad band transducers. 


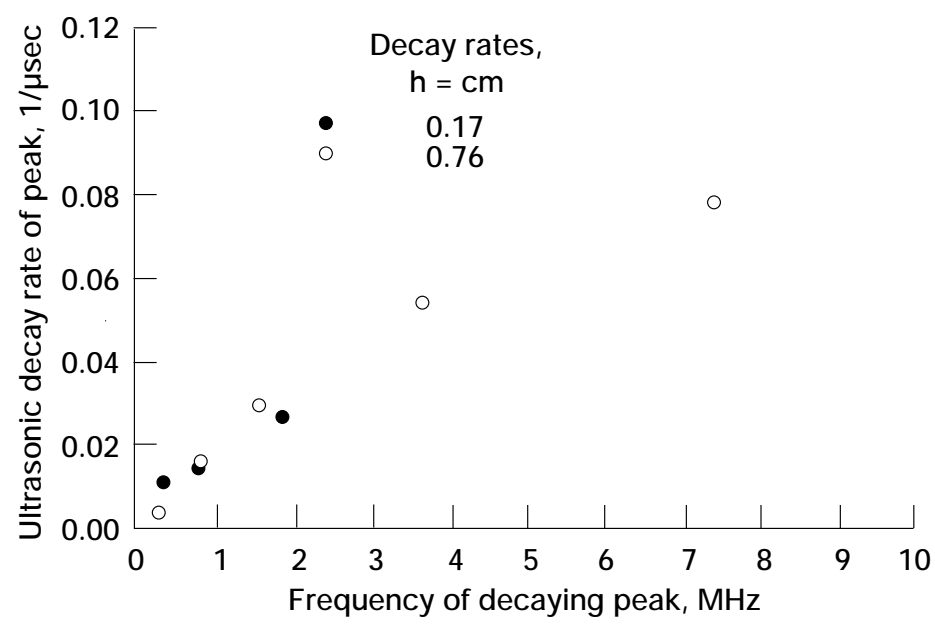

Figure 9.-Acousto-ultrasonic decay versus center frequency of the power spectrum partition for the thick and thin undirectional SCS-6/Ti 15-3, wave path [0].

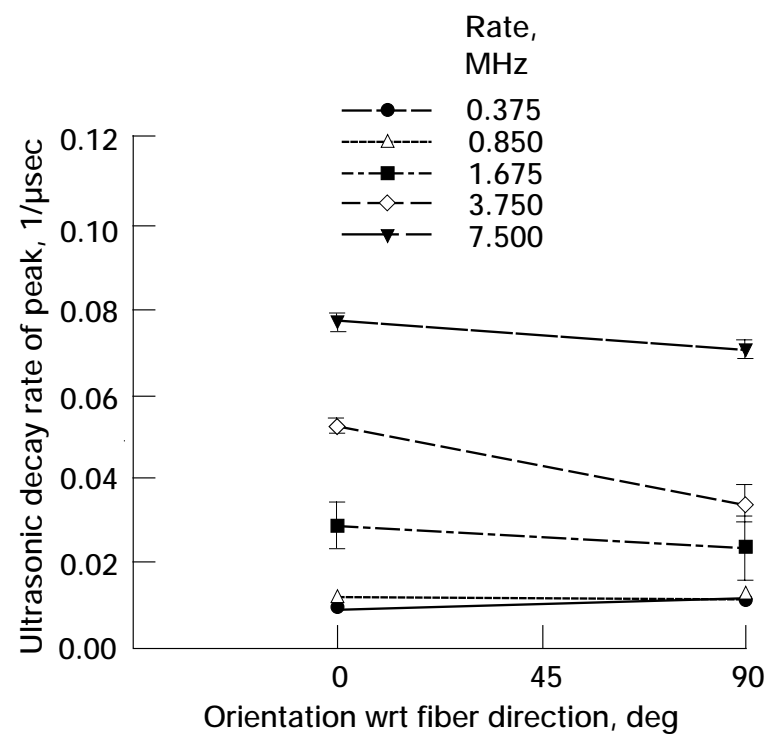

Figure 10.-Acousto-ultrasonic decay versus wave path orientation for the thick and thin unidirectional SCS-6/Ti 15-3. 


\section{APPENDIX \\ Choice of Time and Frequency Partitions for Ultrasonic Decay \\ Calculations}

An example calculation is performed here on a waveform collected from the thin, $(\mathrm{h}=0.17 \mathrm{~cm})$, MMC panel using 2.25 MHz broad band transducers. The waveform is shown in figure 6.

In the UD calculation, time domain partitions are constucted first, followed by power spectra of these partitions. The partitioning strategy is decided by first looking at the overall amplitude spectrum. This is shown in figure 1a. This spectrum has a large peak at $1.875 \mathrm{MHz}$, which is typical of the $2.25 \mathrm{MHz}$ transducers with elastomer coupling pads. The $1.875 \mathrm{MHz}$ peak dominates this waveform. The ratio is:

$$
\lambda / \mathrm{h}=2.1
$$

This catagorizes the waveform as regime 2. $1.875 \mathrm{MHz}$ is approximately the through-thickness resonance fundamental frequency. (The series of lesser peaks are harmonics).

The power spectrum filter 1.5 to $2.5 \mathrm{MHz}$ is chosen. Figures $2 \mathrm{a}, 3 \mathrm{a}$, and $4 \mathrm{a}$ show graphs of log-power spectrum for 1.5 to $2.5 \mathrm{MHz}$ plotted against the midpoint of the time partitions for which they have been determined. Figures $2 \mathrm{a}$ to $4 \mathrm{a}$ differ in the fineness of the time partition grid. Regression results for the different grid widths are shown below:

Figure 2a: grid width $=20 \mu \mathrm{sec}$., Decay rate=.02605/ $\mu$ sec., $\mathrm{R}=0.99$

$$
\begin{array}{lll}
3 \mathrm{a}: & =5 \mu \mathrm{sec} ., & =.02484 / \mu \mathrm{sec} ., \mathrm{R}=0.85 \\
4 \mathrm{a}: & =2 \mu \mathrm{sec} ., & =.02497 / \mu \mathrm{sec} ., \mathrm{R}=0.72
\end{array}
$$

Each of the regressions were performed starting with the first point beyond $40 \mu \mathrm{sec}$. in the waveform record. This avoids the initial rise, typical of A-U waveforms. The regressions were terminated by $140 \mathrm{MHz}$, where it was judged that the signal to noise ratio had become too low. Note that as the grid width is increased the apparent regression correlation coefficient, R, also increases. This is because more of the noise fluctuation is averaged out before the regression with wide grids. Narrow grid results are likely as accurate as wide grid, but the averaging over fluctuations occurs during the regression rather than before it.

The frequency filter of 1.5 to $2.5 \mathrm{MHz}$ was chosen in order to window the fundamental peak at $1.875 \mathrm{MHz}$. It is possible to do a UD rate calculation windowing the harmonics also. The precision is lower because the peaks are a small portion of the total signal. Nevertheless the $3.7 \mathrm{MHz}$ peak gave UD rates in the range of .03 to $.05 / \mu$ sec. while the 5.3 $\mathrm{MHz}$ UD rates gave .05 to $.07 / \mu \mathrm{sec}$. These values agree well with those shown in figure 9 for very different experimental conditions. 
A useful rule-of-thumb is that the exponential decay region of the time domain should cover at least two orders of magnitude of the power spectrum. When this is so the linear portion of the log power spectrum will range over the natural $\log (100)$, or about 4.6. (When setting up the experiment one may approximate this by assuring that the amplitude spectrum covers one order of magnitude). This may be realizable by increasing input power at the sending transducer or decreasing transducer spacing. These strategies will increase the signal-to-noise ratio and lengthing the exponenial decay region.

In order to employe UD in materials studies one must anticipate comparing measurements of different values of UD rates. This might include variations in fabrication parameters between specimens, before and after thermal or mechanical degradation on the same specimen, or some other combination. In any case, it is necessary to be able to validly compare UD rates. Since UD rates are a function of frequency, rates must be compared for the same power spectrum partitions. It probably is not necessary that the input power be the same since changes with time are being compared rather than absolute values. If the "after" UD rate is higher than the "before" rate then it will cover a shorter time interval for the same change in power spectrum. It is likely to be more important that the power spectrum drop by the same factor than it is for the time interval to remain constant.

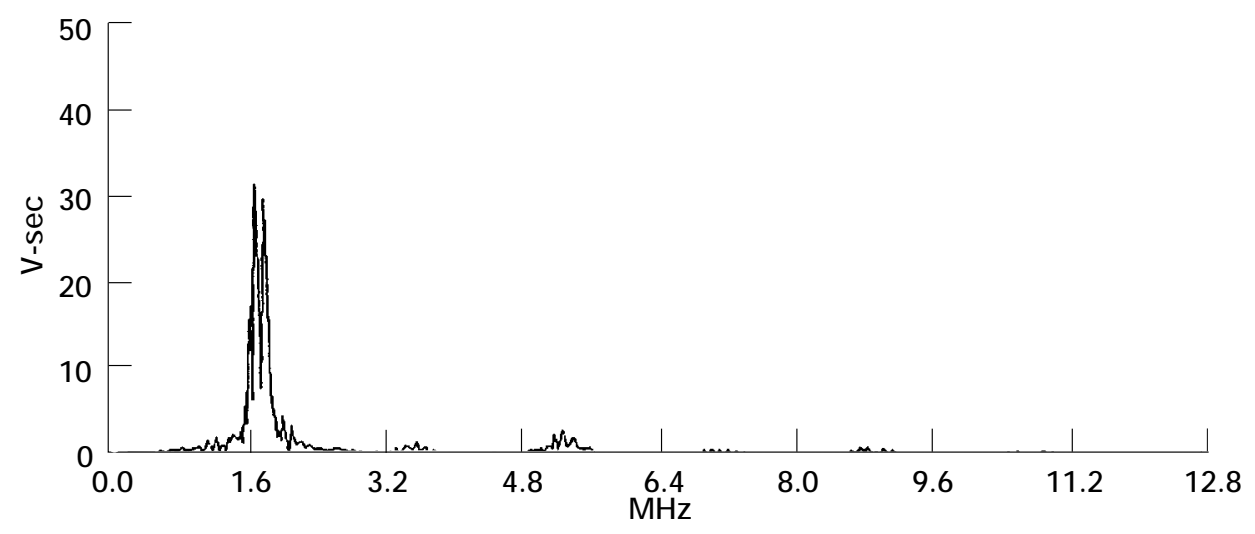

Figure 1a._A magnitude spectrum for waveform of Figure 6.

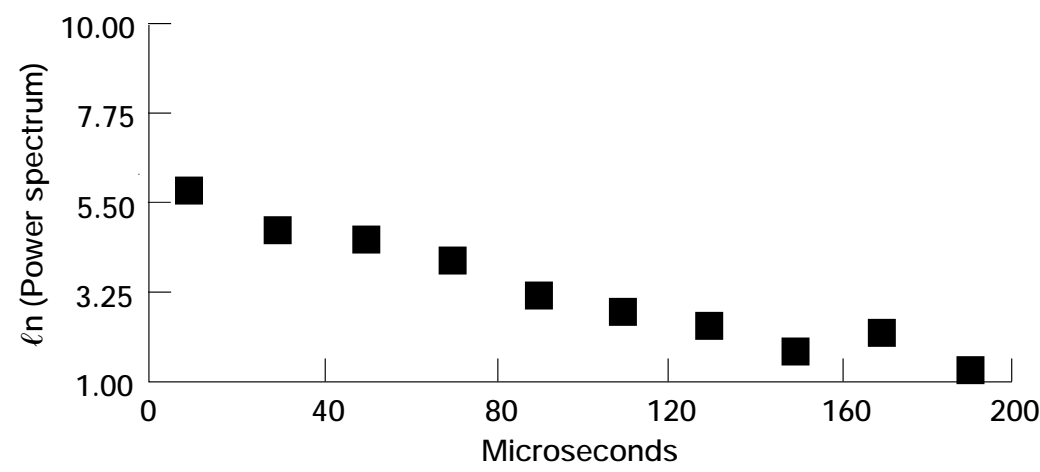

Figure 2a.-Semi-log plot of power spectrum of the 1.5 to $2.5 \mathrm{MHz}$ filter vs center of time partitions $\delta \mathrm{t}=20 \mu \mathrm{sec}$. From the waveform of Figure 6 . 


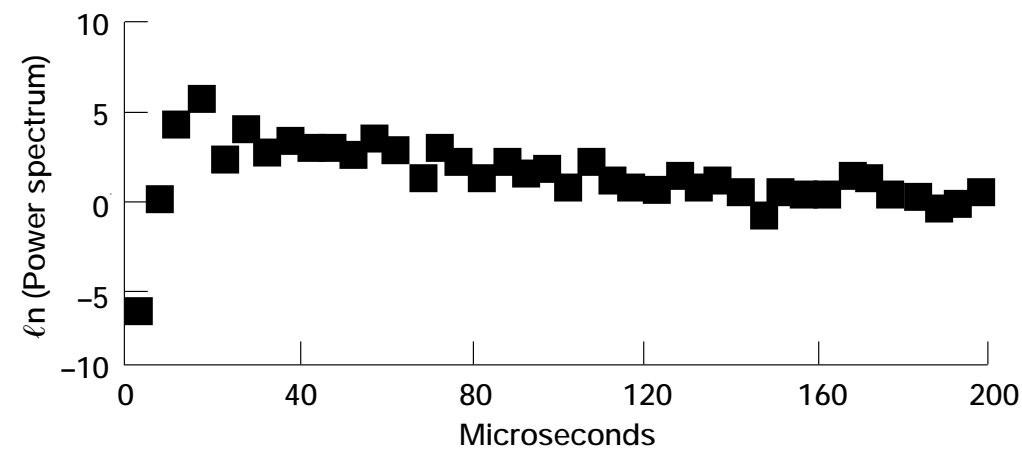

Figure 3a.-Semi-log plot of power spectrum of the 1.5 to $2.5 \mathrm{MHz}$ filter vs center of time partitions $\delta \mathrm{t}=5 \mu \mathrm{sec}$. From the waveform of Figure 6 .

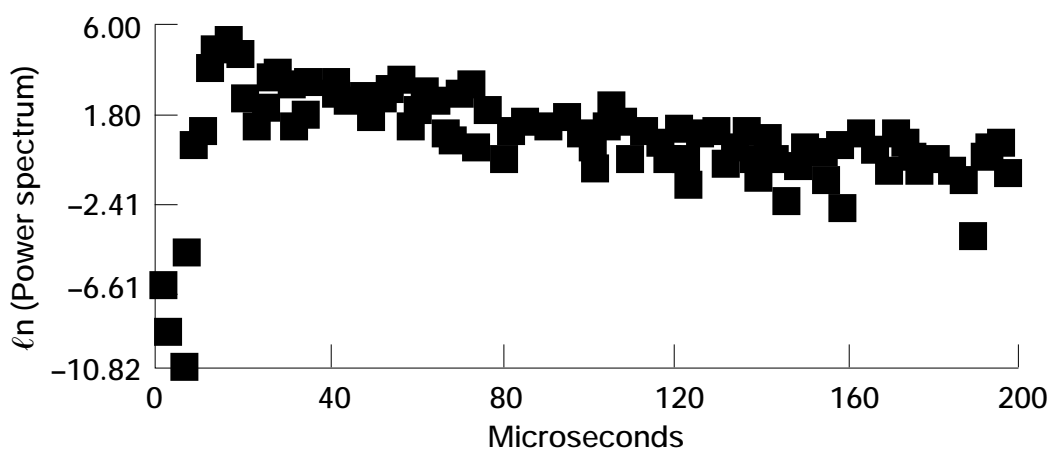

Figure $4 \mathrm{a}$.-Semi-log plot of power spectrum of the 1.5 to $2.5 \mathrm{MHz}$ filter vs center of time partitions $\delta \mathrm{t}=2 \mu \mathrm{sec}$. From the waveform of Figure 6 . 


\section{References}

1. R.L Weaver.: "Diffuse Field Decay Rates For Material Characterization”, Solid Mechanics Research for Quantitative Nondestructive Evaluation. J.D. Achenbach and Y. Rajapaskie, eds., Martinus Nijhoff Publishers, Netherlands, 1987, pp. 425-434.

2. L.A Lott and D.C Kunerth.: "NDE of Fiber-Matrix Bonds and Material Damage in Ceramic/Ceramic Composites", Presented at the American Society of Non-Destructive Testing, Conference on Nondestructive Evaluation of Modern Ceramics, Columbus OH, July 9-12, 1990.

3. H.E. Kautz.: "Determination of Plate Wave Velocities and Diffuse Field Decay Rates With Broad-Band AcoustoUltrasonic Signals", Second International Conference on Acousto-Ultrasonics, ASNT, Atlanta Georgia, June 24-25, 1993, (NASA TM-106158).

4. A Vary.: "The Acoust-Ultrasonic Approach”, First International Conference on Acousto-Ultrasonics, AcoustoUltrasonics: Theory and Application, cosponsored: NASA and ASNT, Blacksburg, VA, 1987, (NASA TM-89843).

5. J.M Periera, H.E. Kautz, D.J. Roth and J. Duke Jr.: "Vibration and Acousto-Ultrasonic NDE for Monitoring Mechanical Damage in Ceramic Matrix Composites”, Presented at the Fourth Annual Research Symposium, of the ASNT Spring Conference, Las Vegas, NV, March 20-24, 1995.

6. B. Tang and E.G Henneke II.: "Long Wavelength Approximation for Lamb Wave Characterization of Composite Laminates", Res. Nondestr. Eval.,Vol. 1, 1989, pp. 51-64.

7. H.E. Kautz: "Detecting Lamb Waves with Broad-Band Acousto-Ultrasonic Signals in Composite Structures", Nondestr. Eval., Vol. 4, 1992, pp. 151-164, (NASA TM-105557).

8. J.H. Williams Jr., H. Karagulle and S.S. Lee: “Ultrasonic Input-Output for Transmitting and Receiving Longitudinal Transducers Coupled to Same Face of Isotropic Elastic Plates”, Mat. Eval. Vol. 40, 1982, pp. 655-662.

9. H.E Kautz.: "Ultrasonic Evaluation of Mechanical Properties of Thick, Multilayered, Filament-Wound Composites", Mater. Eval., Vol. 45, 1987, pp. 1404-1412.

10. H.E.Kautz and B.A. Lerch: "Preliminary Investigation of Acousto-Ultrasonic Evaluation of Metal-Matrix Composite Specimens", Mater. Eval. Vol. 49, 1991, pp. 607-612.

11. H.E. Kautz and R,T, Bhatt: "Ultrasonic Velocity Technique for Monitoring Property Changes in Fiber-Reinforced Ceramic Matrix Composites”, Ceram. Eng. Sci. Proc., Vol. 12, 1991, pp. 1139-1151, (NASA TM-103806).

12. D.J. Roth, et al.: "Review and Statistical Analysis of the Ultrasonic Velocity Method for Estimating the Porosity Fraction in Polycrystalline Materials", NASA TM-102501, 1990. 


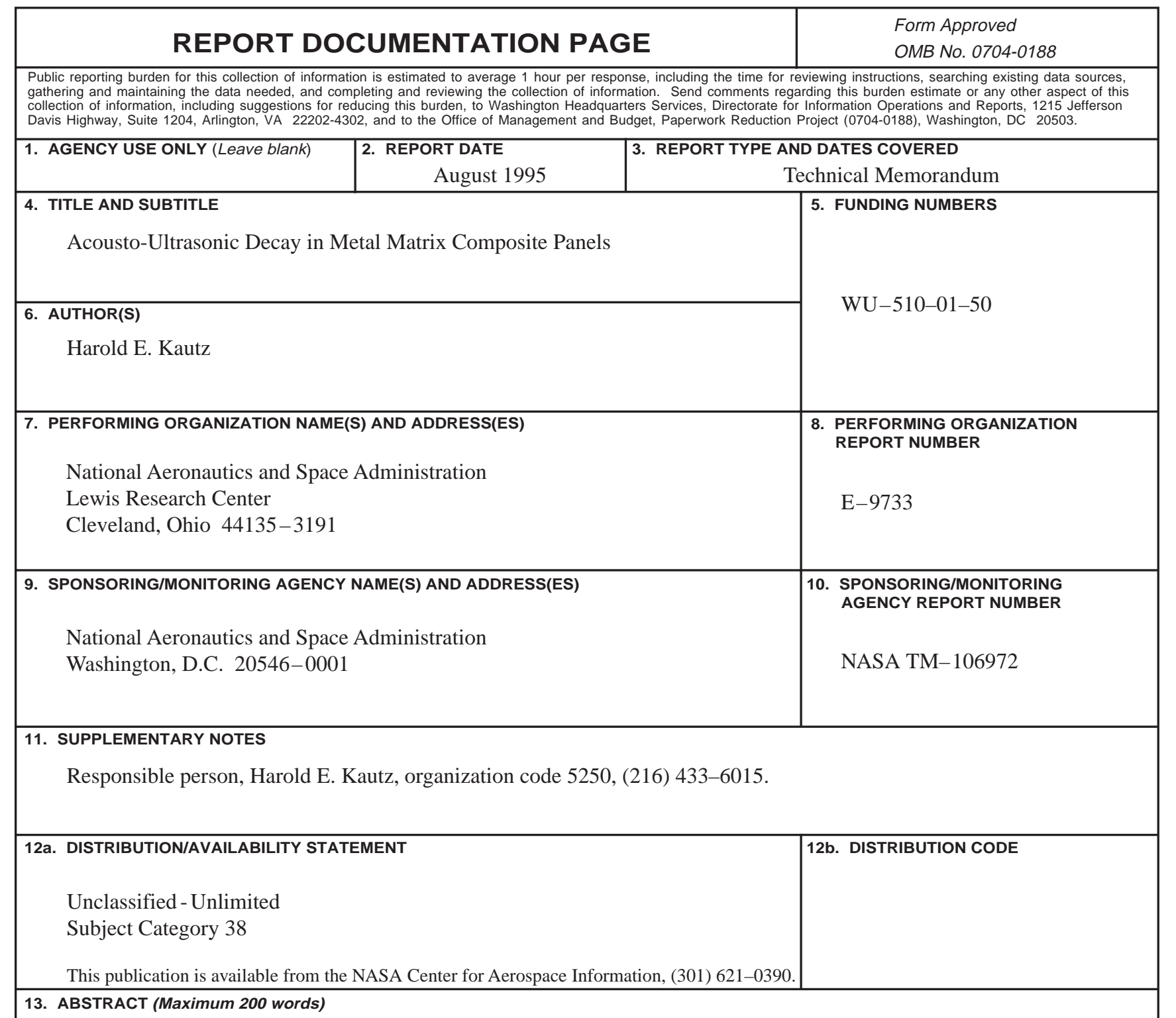

Acousto-ultrasonic (A-U) decay rates (UD) were measured in metal matrix composite (MMC) panels. The MMC panels had fiber architectures and cross-sectional thicknesses corresponding to those designed for aerospace turbine engine structures. The wavelength-to-thickness ratio produced by the combination of experimental frequency setting conditions and specimen geometry was found to be a key parameter for identifying optimum conditions for UD measurements. The ratio was shown to be a useful rule of thumb when applied to ceramic matrix composites (CMC)s and monolithic thermoplastics.

\begin{tabular}{|l|l|}
\hline $\begin{array}{l}\text { 14. SUBJECT TERMS } \\
\text { Nondestructive tests; Ultrasonics; Ultrasonic tests; Ceramics; } \\
\text { Ceramic matrix composites; Metal matrix composites }\end{array}$ \\
\hline $\begin{array}{c}\text { 17. SECURITY CLASSIFICATION } \\
\text { OF REPORT } \\
\text { Unclassified }\end{array}$ & $\begin{array}{c}\text { 18. SECURITY CLASSIFICATION } \\
\text { OF THIS PAGE } \\
\text { Unclassified }\end{array}$ \\
\hline NSN 7540-01-280-5500 &
\end{tabular}

